EOMmun Communication et organisation

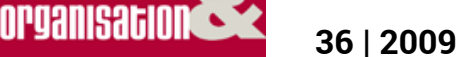

Pour une approche communicationnelle de l'individu au travail

\title{
Pour une approche communicationnelle de l'individu au travail
}

Laurent Morillon, Arlette Bouzon et François Cooren

\section{CpenEdition}

\section{Journals}

Édition électronique

URL : http://journals.openedition.org/communicationorganisation/874

DOI : 10.4000/communicationorganisation.874

ISSN : $1775-3546$

Éditeur

Presses universitaires de Bordeaux

Édition imprimée

Date de publication : 1 décembre 2009

Pagination : 7-18

ISBN : 978-2-86781-719-9

ISSN : $1168-5549$

Référence électronique

Laurent Morillon, Arlette Bouzon et François Cooren, « Pour une approche communicationnelle de l'individu au travail », Communication et organisation [En ligne], 36 | 2009, mis en ligne le 19 avril 2011, consulté le 10 décembre 2020. URL : http://journals.openedition.org/communicationorganisation/874 ; DOI : https://doi.org/10.4000/communicationorganisation.874 


\title{
Pour une approche communicationnelle de l’individu au travail Laurent Morillon, Arlette Bouzon, François Cooren
}

\author{
laurent.morillon@iut-tlse3.fr \\ arlette.bouzon@iut-tlse3.fr \\ f.cooren@umontreal.ca
}

L’actualité qui a accompagné la conception de ce numéro 36 de la revue Communication \& Organisation montre combien le travail - $a$ fortiori lors de périodes de tensions organisationnelles médiatisées est susceptible d'occuper une grande place dans la vie de l'individu : revendications et grèves suite à des délocalisations, des fermetures de sites (Molex, Continental...) et/ou des licenciements massifs (Moulinex, Caterpillar, Freescale...), séquestrations de dirigeants, destructions de l'outil de travail ${ }^{1}$, témoignages de souffrance au travail, suicides (à Thalès, Renault, France Télécom...). Plus encore qu'une activité au sens extensif du terme (au croisement de la technique, de l'économie et de l'organisation sociale), le travail est à considérer comme l'un des principes fondateurs de la vie sociale : il est l'un des principaux lieux d'échanges entre les individus et l'un des modes essentiels de réalisation de soi, de construction identitaire et de socialisation "secondaire" au sens de Berger et Luckmann (1966). Les univers professionnels conduisent en effet à l'intériorisation de sous-mondes institutionnels spécialisés et contribuent à la production d’une identité spécifique (Causer, Gasparini, Durand, 2009).

La diversité des discours parfois caricaturaux sur le rapport des Français à leur travail symbolise bien ce qui se peut se jouer dans les mondes professionnels. De manière relativement schématique, à l'opposé l'un de l'autre, deux types de discours se confrontent, voire s'affrontent, sur les scènes organisationnelle, littéraire et médiatique. À l'un des extrêmes, les modes de management actuels, ainsi que le rapport et l'investissement au travail, sont explicitement critiqués. Ces discours sont alimentés entre autres par des livres à succès comme Souffrance en France de C. Dejours (2000) ou Bonjour Paresse de C. Maier (2004), des films comme Danger travail de P. Carles (2003),

1 Pour Alter (2009), à la suite des travaux de Mauss (1924), l'avènement de la violence est en partie expliqué par le refus du don. 
Dossier : Pour une approche communicationnelle de l'individu au travail

des documentaires comme La mise à mort du travail de J.-R. Viallet (2009), des articles de journalistes, des rapports d'analyse sur le " bien-être au travail », dont celui du Cabinet Technologia ou encore le baromètre de l'Agence Nationale pour l'Amélioration des Conditions de Travail (ANACT). À l'opposé de ces discours, ceux issus de l'entreprise et plus particulièrement des services de ressources humaines prônent l'investissement de l'individu au travail, la flexibilité, la mobilisation des «collaborateurs " ${ }^{2}$ et de leurs compétences pour une plus grande efficacité, le maintien du concours actif et de la motivation d'un personnel déclaré depuis les années 1990 comme un « capital précieux » (Weil, 1990).

Entre ces divers discours parfois passionnés, les coordinateurs de ce numéro ont estimé qu'il existait une place pour une approche et une lecture scientifique, distanciée, portant sur le rapport et la place de l'individu au travail. Mais qu'est-ce qu'un «individu » ? En quoi estil différent d'un «acteur» ou d'un «agent» ? Penser l'individu implique en fait de pouvoir dépasser l'opposition entre liberté et déterminisme. Car dans une pensée complexe, ce terme ${ }^{3}$ exige la prise en compte d'une pluralité de dimensions : il est tout à la fois un sujet économique qui tente de valoriser ses biens ; un sujet juridique pourvu de droits et de devoirs ; un sujet éthique auquel est dû un respect inconditionnel et qui le manifeste à autrui ; un sujet philosophique capable d'autoréflexion et plus largement de rationalité. Elias (1973 ; 1975) dans une sociologie historique, Macpherson (2004) ou Taylor (1998), à partir d'une étude de la pensée, ont participé à décrypter, dans le sillage des analyses classiques de Marx, Durkheim, Weber et Simmel ce long processus socio-historique et ont resitué l'individu dans des configurations de relations sociales, récusant la fiction d'un individu autosuffisant. S'écartant d'une conception de l'individu orienté par ses intérêts et ses intentions privées, le terme d'acteur est, quant à lui, caractérisé depuis Parsons (1960) par la manifestation de conduites sociales personnelles et collectives, reflétant des assignations de rôles et de statut : l'acteur joue des rôles imposés par une place sociale sans autonomie face à une société régie par les

\footnotetext{
${ }^{2}$ Voir à ce sujet l'article de J-L. Bouillon et E. Maas dans ce dossier.

${ }^{3}$ Y. Andonova et B. Vacher dans leur article estiment que l'individu est une personne autonome, c'est-à-dire qui a intégré les contraintes (règles, représentations et marquages sociaux) qui la dépassent pour les surmonter et ainsi être capable de les mobiliser alors que le sujet serait l'individu émancipé, capable de se libérer des contraintes, par une forme de méditation ou de sagesse, pour les transformer en œuvre.
} 
Dossier : Pour une approche communicationnelle de l'individu au

travail

impératifs du pouvoir et du profit. Clairement impliqué dans une relation de subordination il s'est cependant vu reconnaître depuis Crozier et Friedberg (1977) la possibilité de choix rationnels et une capacité d'opposition aux contraintes du système. Cette évolution le fait se rapprocher in fine de la définition d'agent inspirée des travaux de Giddens (1984) : les êtres humains sont des agents connaissants, même s'ils agissent à l'intérieur des limites historiquement spécifiées que posent des conditions sociales qu'ils ne reconnaissent pas et des conséquences de leurs actes qu'ils ne peuvent prévoir. Finalement, avec Touraine (2007), ce rapprochement de définitions des termes «acteur » et «sujet ", va jusqu'à la production d'une théorie d'un sujet qui intègre les contraintes de la mondialisation, marque sa volonté de liberté, affirme sa singularité et revendique son désir de reconnaissance pour soi et les autres.

\section{L'individu au travail ?}

$\mathrm{Au}$ travail, l'individu intègre des organisations, notamment des entreprises, confrontées depuis les années 1970 à un contexte de mondialisation, à une concurrence nationale et internationale ${ }^{4}$, à une globalisation des secteurs d'activités, à une exigence financière croissante de l'actionnariat ainsi qu'à des crises financière et économique récurrentes. Engagées dans une course à la productivité, ces organisations adaptent leurs structures et leurs formes: élargissement et déconcentration productive (éclatement en petites unités de production, via les techniques d'impartition et structuration en réseaux), « orientation client », filialisation, délocalisation, externalisation, sous-traitance... Les mutations induites de la relation d'emploi (flexibilité, employabilité, précarité...), la fragilisation des collectifs de travail, les nouveaux modes de management ${ }^{5}$, l'augmentation croissante de l'intensité du travail (dont le culte de l'urgence) ainsi que l'émergence de nouveaux types organisationnels (matriciel, par projet...), la prolifération des TIC (créant entre autres

\footnotetext{
${ }^{4}$ Présentant un potentiel de confrontation culturelle « violente » lorsque cette mise en concurrence fait suite à une privatisation et/ou une désinstitutionalisation.

${ }^{5}$ Mise en concurrence, modulation individualisée dans un double mouvement engageant autonomisation et responsabilisation finalisé par des dispositifs d'évaluation et de rémunération variable. Ces dispositifs d'incitations et de sanctions ont aussi des effets pervers. Ainsi, le système de concurrence par primes et pénalités mesurant les performances relatives et respectives peut amener à saboter méthodiquement le travail des collègues (Beauvallet, 2009).
} 
Dossier : Pour une approche communicationnelle de l'individu au travail

des effets de « laisse électronique »)... font des individus plus isolés mais paradoxalement dépendants, multi-engagés et susceptibles de subir de multiples injonctions paradoxales.

Comment dans ces nouveaux modèles productifs les individus se construisent-ils en tant que sujets, évitant d'être réduit à de purs objets ou de purs consommateurs ? Comment l'individu aspirant à l'épanouissement et à la réalisation au travail, crée-t-il et intègre-t-il finalement des dispositifs de soumission librement consentie ? Quelles sont la nature et la portée des nouvelles structures d'organisation ainsi que des dispositifs de pouvoir actuels? Dans le contexte de l'individualisation des rapports sociaux dans les organisations, comment s'articulent l'identité de classe, l'identité professionnelle et les modes plus élargis d'identification ? Au regard des transformations récentes du travail dans le capitalisme contemporain, quels sont finalement la place, le statut et la considération de l'individu au travail ?

Estimant, dans une perspective interprétativiste, que les processus de communication - qu'ils soient médiatisés ou interpersonnels, commandités ou spontanés - sont instituants et constituants des organisations, les coordinateurs ont proposé un appel à article invitant les auteurs à aborder ces questions dans une problématique communicationnelle, ou qui verrait l'objet « communication » occuper une place centrale. Cet appel a suscité un grand intérêt : plus d'une cinquantaine de contributeurs de disciplines différentes ont proposé un résumé initial. Les dix communications retenues ont été sélectionnées d'abord sur cette base, puis à partir d'un texte intégral présenté anonymement à l'expertise. Une démarche constructive de relectures et d'améliorations plus ou moins substantielles a enfin permis la constitution du présent dossier. Ce numéro pluridisciplinaire (comportant des textes en sciences de l'information et de la communication bien sûr mais aussi en sociologie, psychosociologie, psychodynamique du travail) rassemble des articles qui font état de recherches conduites dans des directions distinctes, dont les objets, cadres d'analyse ou démarches de recherches sont pluriels. En prenant comme fil directeur l'individu dans les mondes du travail, les auteurs, pour certains à partir d'enquêtes de terrain, se sont interrogés sur la place et/ou le rôle de la communication dans les transformations de l'organisation et sur la considération de l'individu dans son activité professionnelle. Les concepts d'identité et de reconnaissance sont ainsi devenus des catégories d'analyse discutées. Ces articles permettent d'éclairer de manière complémentaire le réel et les problématiques originales dans les rapports entre le travail, l'individu, les techniques, 
Dossier : Pour une approche communicationnelle de l'individu au

travail

l'organisation voire les institutions. Ils décrivent et favorisent la compréhension de certaines des nouvelles formes que prend le travail aujourd'hui, certaines de ses conséquences pour l'individu et la place qu'occupe la communication dans ces processus en évolution.

Afin de présenter la complexité des phénomènes abordés, nous avons choisi d'organiser les textes retenus selon trois axes. Le premier d'entre eux se veut un constat des différentes formes d'expression de la souffrance au travail (stress, plainte, suicide...). Afin d'éclairer et de contextualiser, en partie du moins, les phénomènes précédemment exposés, les deuxième et troisième axes présentent et analysent respectivement des dispositifs managériaux et de communication à l'oeuvre. Ces analyses scientifiques ont pour objectif de saisir le travail en actes, la question du comment l'emportant sur celle du pourquoi.

\section{L'individu et la souffrance au travail}

Si la souffrance au travail n'est pas en soi un phénomène nouveau, son expression, sa médiatisation et son étude le sont davantage. La question de la santé au travail fut aux débuts de la révolution industrielle au cœur des interrogations sur l'organisation du travail. Elle a servi de point d'appui au développement de l'activité syndicale et de fondement au développement de la protection sociale. Elle revient aujourd'hui avec une acuité renouvelée, notamment par la médiatisation des suicides au travail dans des entreprises comme Renault ou France Télécom. Si le lien entre santé et travail est complexe, ce dernier est dans ses formes contemporaines potentiellement accusé de porter atteinte à la santé physique mais aussi mentale (dépression...). Car au-delà des formes traditionnelles de pénibilité, de nouvelles gênes apparaissent telles que la surexposition au stress et/ou l'accroissement de la charge cognitive dans l'activité. Les indicateurs des risques au travail sont d'ailleurs à la hausse, qu'il s'agisse des accidents du travail depuis 1995 (1,2 million par an), des maladies professionnelles déclarées, des troubles musculo-squelettiques, des cancers professionnels (source CNAMTS $^{6}$ ). Le dernier baromètre de l'Agence Nationale pour l'Amélioration des Conditions de Travail constate ainsi que $64 \%$ des salariés portent les stigmates de leur malaise professionnel : fatigue,

6 La Caisse Nationale de l'Assurance Maladie des Travailleurs Salariés (CNAMTS) est un établissement public national à caractère administratif, jouissant d'une personnalité juridique et d'une autonomie financière. 
Dossier : Pour une approche communicationnelle de l'individu au travail

tensions, insomnies, irritabilité. Ajoutons que les inégalités sociales exposent davantage encore certaines populations (ouvriers ${ }^{7}$, femmes, jeunes, immigrés...) à ces phénomènes. Les trois textes de ce premier axe présentent et questionnent donc cette expression de la souffrance auprès de différents publics. Marc Loriol compare ainsi deux groupes professionnels - policiers et infirmières - dont le travail est réputé stressant. Il montre comment les échanges informels dans et sur le travail n'ont pas seulement pour effet d'apporter informations et réconfort mais participent également à la production collective du sens de l'engagement et finalement de l'expérience du stress. Il met en évidence l'importance des échanges sur le travail entre collègues pour faire face aux situations pénibles. Ceux-ci permettent de construire collectivement un sens acceptable pour les événements à l'origine des émotions négatives.

Olivia Foli s'intéresse, pour sa part, dans le cadre d'une étude ethnographique menée dans une organisation bureaucratique, à l'expression du mal-être à travers les paroles de plainte. Celle-ci, construite socialement et régulée par le milieu où elle s'exprime, est dans certains cas disqualifiée. Le plaignant ne peut alors témoigner d'un motif de mal-être. Il perçoit que ses ressentis et ses demandes sont difficilement avouables à sa hiérarchie et même à ses pairs à moins de les exprimer sous une forme très feutrée, ne risquant pas de heurter son auditoire.

Pascale Molinier présente enfin les résultats d'une recherche particulièrement riche menée en psychodynamique du travail à la suite du suicide d'un jeune cadre. Cette recherche essaye de comprendre quelles sont les incidences de cette mort tragique sur les autres membres des équipes et sur leur vécu au travail. Prenant en considération la forte charge émotionnelle propre à un tel geste de désespoir ultime, Pascale Molinier analyse l'isolement des individus dans leur rapport aux risques ainsi que les phénomènes de rumeur et leurs capacités d'occultation. Elle convoque notamment le concept de «stratégie collective de défense ».

\section{L'individu et le management}

Le deuxième axe de ce dossier présente et analyse certains des dispositifs managériaux à l'œuvre dans les organisations modernes et

7 La France compte six millions d'ouvriers. Or, à l'âge de 35 ans, un cadre peut espérer vivre sept ans de plus qu'un ouvrier, et dix ans de plus sans incapacité (cf. Debout F. et al. 2009). 
Dossier : Pour une approche communicationnelle de l'individu au

travail

ce à deux niveaux : « méta » et « micro ». Au premier de ceux-ci, de manière surplombante donc, l'action managériale consiste en la prise de décisions structurantes (division du travail, hiérarchisation des activités...) par des directions générales (organisation, ressources humaines...). Ces choix engageants peuvent être influencés par des tendances qui transcendent les organisations (équipes projet, ERP, «bonnes pratiques »...). Or, face au travail qui s'affranchit des cadres et des temporalités classiques, la coordination des individus au travail devient un phénomène plus fuyant, plus difficile que jamais à appréhender. Deux textes s'intéressent à cette dimension « méta » du management. Tout d'abord, Jean-Luc Bouillon et Elise Maas étudient l'usage d'un terme symbolique, celui de «collaborateur», qui s'intègre dans la projection d'une situation enchantée et idéalisée des relations sociales. Les stratégies individuelles, l'asymétrie des rapports sociaux, les divergences d'intérêts sont passées sous silence au profit de la description performative d'une organisation cohérente, unitaire et fluide où chacun coopère. L'étude conduite au sein d'une société internationale de services informatiques met ainsi en évidence la manière dont cette figure idéalisée du collaborateur relève de rationalisations communicationnelles, elles-mêmes articulées à des rationalisations productives plus générales, impliquant la transformation des processus productifs et commerciaux et le développement d'outils informatiques.

Claire Burlat étudie, quant à elle, les nouveaux rapports au travail induits par l'implantation d'un modèle unifié de gestion des personnels dans la maison mère et les filiales d'une multinationale, sans prise en compte des cultures concernées (organisationnelle, nationale...). Avec l'emprunt d'un objet d'étude propre aux sciences de gestion (les transferts de pratiques de management), elle tente de comprendre un phénomène social du point de vue des processus communicationnels. Son article montre leur diversité au travers de l'analyse comparée de trois situations de transferts de pratiques managériales au sein d'un même groupe international de services. Elle met en évidence la diversité des formes prise par la négociation sociale et son influence sur la transformation des modes de socialisation professionnelle.

À un niveau « micro », ce sont les pratiques des managers eux-mêmes qui sont prises en considération. L'introduction et la mise en oeuvre d'outils de gestion peuvent en effet également s'interpréter comme la rencontre plus ou moins antagoniste entre un manager et ses subalternes mais aussi comme l'expression d'un conflit de rationalités. Informer et communiquer étant des vecteurs privilégiés pour marquer 
Dossier : Pour une approche communicationnelle de l'individu au travail

la considération et la reconnaissance, de nombreux instruments participent de la mise en visibilité de l'individu au travail (rapports d'activité, organigramme, entretiens d'évaluation, fiches de poste...). Hier relativement préservés, ces managers se trouvent également aujourd'hui particulièrement exposés : autonomes quant aux manières d'atteindre leurs objectifs annualisés, ils sont aux prises avec des équipiers plus exigeants et des dispositifs destinés à améliorer leurs performances (cf. le succès de pratiques qu'il est convenu d'appeler le coaching, le relooking ou l'évaluation à $360^{\circ} \ldots$..). 28\% des cadres présenteraient un niveau de stress aigu (Combalbert, RiquelmeSénégou, 2006). Marie France Peyrelong a ainsi mené une enquête auprès des cadres intermédiaires dans les bibliothèques sur la manière dont ils perçoivent les manifestations de reconnaissance sur le lieu de travail. Les interlocuteurs interrogés montrent combien les organisations au quotidien peuvent rester silencieuses vis-à-vis de certaines contributions pourtant indispensables au bon fonctionnement mais sans visibilité extérieure (par exemple l'assistance à un collègue, des compromis lors du passage aux 35 heures, etc.). Ces réflexions nous renvoient à d'autres travaux (Giroux, 2006), car si l'activité est mise en forme et fait l'objet de reporting, la question de la reconnaissance de l'activité d'un individu, voire de l'individu luimême, demeure.

Dans cette même veine, Valérie Lépine a, quant à elle, porté son attention sur les cadres de santé, cadres de proximité qui mobilisent de multiples ressources communicationnelles pour engager un processus de reconnaissance à l'égard des équipes dont ils ont la responsabilité, là où semble-t-il, le soutien de l'organisation fait le plus souvent défaut $^{8}$. Le secteur hospitalier est concerné depuis vingt ans par de profondes réorganisations. Il a fait l'objet de travaux scientifiques d'une grande qualité (dont Grosjean, Lacoste, 1999) régulièrement renouvelés. Ceux-ci ont mis au jour l'importance des interactions quotidiennes dans l'activité et des ajustements multiples nécessaires à l'accomplissement des tâches dans un contexte où la pression subie et le stress des personnels n'ont cessé de s'accentuer.

\footnotetext{
${ }^{8}$ Les articles publiés dans le présent dossier mettent aussi en évidence des formes de solidarité qui soutiennent les collectifs de travail et explorent comment la foule des " objets intermédiaires » (Jeantet, 1998) équipent la coordination des professionnels entre eux. Certaines activités apparaissent ainsi moins verticales et hiérarchiques que transversales et réticulaires, autorisant en temps réel la combinaison de ressources sociales et matérielles. La place des échanges informels y est centrale.
} 
Dossier : Pour une approche communicationnelle de l'individu au

travail

\section{L'individu et la communication}

Dans le troisième et dernier axe de ce dossier, ce sont les pratiques, situations, processus et dispositifs de communication qui sont étudiés pour éclairer la place, le statut ou encore la considération de l'individu au travail. La communication dans la sphère du travail est multiple, diverse et fondamentalement complexe. Elle fait le quotidien des organisations qui sont à considérer comme des ensembles semi autonome de relations qui prennent leurs origines dans les interactions humaines (Bouzon, 2010). La réalité de ces systèmes organisationnels dynamiques et interactifs, est donc co-construite par les acteurs euxmêmes.

Thomas Heller s'interroge sur les implications assujettissantes de pratiques et situations de communication en entreprise. À partir des travaux de Foucault, il cherche à appréhender la nature profonde du pouvoir, à la fois stratégie globale, interactions locales et dynamiques d'expansion. Il montre que la définition des dispositifs de gestion incarne les représentations dominantes et oriente, canalise l'activité de chacun, en contribuant à la perpétuation des rapports de domination. Tout en replaçant l'étude dans les acquis de la sociologie du travail, sa réflexion permet de ne pas raisonner seulement en terme d'effets socio-organisationnels mais également en prenant en compte les interactions dissymétriques entre un système (principes, normes, outils) et des acteurs, ou plus exactement entre des agents. Il rend ainsi compte de l'inscription des principes de gestion et de l'action managériale dans la production de l'ordre... ou du désordre.

Anne Piponnier traite du rôle du processus de communication dans les organisations en mode projet et particulièrement de leur dispositif médiatique. Partant de l'hypothèse que le projet n'existe pas en dehors de sa mise en scène, elle examine en quoi l'activité de communication est structurante et conduit à renforcer le modèle de la contrainte souple. Son étude empirique s'appuie sur un échantillon de projets de communautés scientifiques (150 sites actifs) et met notamment au jour la tension que chaque acteur subit entre autonomie et rationalisation.

Yanita Andonova et Béatrice Vacher s'interrogent enfin sur les interactions entre acteurs humains et non-humains, là où des dispositifs communicationnels s'entrecroisent avec des dispositifs normatifs. Elles confrontent ainsi deux terrains organisationnels qui, partant de la même question de l'invisibilité des TIC, aboutissent à des conséquences radicalement opposées. Au sein des ateliers de production, ces outils valorisent les utilisateurs en quittant la sphère des objets techniques énigmatiques pour devenir des dispositifs 
Dossier : Pour une approche communicationnelle de l'individu au travail

quotidiens invisibles et provoquer l'estime de la hiérarchie, alors que dans un centre de recherche, les TIC dévalorisent les personnes qui en ont la charge en rajoutant des tâches fastidieuses et invisibles (programmation, classement, tri), omises et dévaluées par la hiérarchie.

\section{Pour conclure}

L'instabilité des situations d'emploi, l'incertitude sur le devenir des entreprises, les relations de travail difficiles entraînent des processus de précarisation avec leurs lots de fragilité, de vulnérabilité et de craintes alors même que perdure "l'attachement " au travail. Mais pour autant et à la lecture des différentes contributions de ce dossier, l'individu ne peut être systématiquement considéré comme une " victime » d'un système susceptible de le «broyer ». Plus diplômés, informés, cultivés et critiques, un certain nombre d'entre eux se révèlent également plus opportunistes, n’hésitant plus à changer d'organisation au gré de projets professionnel et personnel évolutifs. Mieux préparés à l’imprévisibilité du monde du travail, ces individus et notamment ceux de la génération «Y » (Tulgan, Carolyn, 2001), ont conscience qu'ils exerceront différents métiers dans des environnements variés tout au long de leur carrière. Ils disposent pour s’y préparer de mobilités intellectuelle (par la formation tout au long de la vie), sectorielle et géographique. Ils développent également des réseaux et les maintiennent actifs en usant des TIC (via des sites tels Linkedin, Facebook ou encore Twitter). Ils accroissent enfin leur " employabilité » et leurs " compétences », maîtrisant en cela les codes des directions de ressources humaines.

Les recherches présentées dans le présent dossier de la revue Communication \& Organisation démontrent, s'il en était besoin, la complexité des phénomènes à l'œuvre dans les organisations. Car même si la diversité des terrains, des approches, des méthodes et des disciplines des auteurs est susceptible d'apporter un éclairage original sur l'individu au travail, l'entrée par la communication est forcément particulière et parcellaire. Ces contributions participent cependant au renouvellement conceptuel de la discipline: de nombreuses perspectives théoriques ont rejoint, complété, discuté, prolongé, voire critiqué, les modèles dominants, dont il est difficile de rendre compte de manière systématique et détaillée dans ce numéro. Elles devraient toutefois donner les moyens de comprendre certaines des mutations contemporaines des organisations et déboucher sur l'identification d'axes de recherches prioritaires autour de l'individu au travail. Cellesci pourraient alors permettre un renouvellement majeur de la 
Dossier : Pour une approche communicationnelle de l'individu au

travail

problématique concernant la nature, les formes sociales du travail et leurs conséquences.

\section{Bibliographie}

ALTER N. Donner et prendre : la coopération en entreprise. Paris : La découverte, 2009

BERGER, P. L., LUCKMANN T. The Social Construction of Reality: A Treatise in the Sociology of Knowledge. NY : Garden City, Anchor Books, 1966

BEAUVALLET M. Les stratégies absurdes. Paris : Seuil, 2009

BOUZON A. Entre communication et management. Evolution des fondements épistémologiques des organisations. in: II Congreso Internacional de la Asociación Española de Investigadores de la Comunicación (AE-IC), «Comunicación y desarrollo en la era digital », Faculté des sciences de la communication de l'Université de Málaga, 3-5 Février 2010

CAUSER J.-Y., DURAND J.-P., GASPARINI W. Les identités au travail. Toulouse : Octares, 2009

COMBALBERT N., RIQUELME-SENEGOU C. Le Mal-Être au travail. Paris : Presses de la Renaissance, 2006

CROZIER M., FRIEDBERG F. L'acteur et le système : Les contraintes de l'action collective. Paris : Editions du Seuil, 1977

DEBOUT F., FAURE S., FLIPO F., GERNET I., LE LAY S., LUSSON J., et VINCENT J. La santé à l'épreuve du travail. in : Mouvements. 2009/2 - n 58, pp. 7 à 12.

ELIAS N. La Civilisation des mœurs. Paris : Calmann-Lévy, 1973

ELIAS N. La Dynamique de l'Occident. Paris : Calmann-Lévy, 1975

GIDDENS A. La Constitution de la société (1984), trad. AUDET M., Paris : PUF, Quadrige, 2005.

GIROUX N. Le nouage des savoirs en organisation in : BOUZON A. (dir.), La communication organisationnelle en débat: champs, concepts, perspectives. Paris : L'Harmattan, 2006, pp. 159-189

GROSJEAN M., LACOSTE M. Communication et intelligence collective. Le travail à l'hôpital. Paris: PUF, Le Travail Humain, 1999 
Dossier : Pour une approche communicationnelle de l'individu au travail

JEANTET A. Les objets intermédiaires dans les processus de conception des produits. in: Sociologie du travail, 3/1998, pp. 291316.

MACPHERSON C.B. La théorie politique de l'individualisme possessif, De Hobbes à Locke. Paris : Gallimard, Folio, 2004

MAUSS M. Essai sur le don (1924). Paris : PUF, 1968

PARSONS T. Structure and Process in Modern Societies, Glencoe : Free press, 1960

SALENGRO B. Le stress des cadres. Paris : L’Harmattan, 2005

TAYLOR C. Les sources du moi. Paris : Seuil, 1998

TOURAINE A. Penser autrement, Paris : Fayard, 2007

TULGAN B., CAROLYN A. M. Managing Generation Y : global citizens born in the late seventies and early eighties. Amherst : HRD Press, 2001

VATIN F. Le travail et ses valeurs. Paris : Albin Michel, 2008

WEIL P. Communication Oblige. Paris : Les éditions d'Organisation, 1990 